\title{
Norm Estimates for Solutions of Polynomial Operator Equations
}

\author{
Michael Gil’ \\ Department of Mathematics, Ben Gurion University of the Negev, P.O. Box 653, 84105 Beer-Sheva, Israel \\ Correspondence should be addressed to Michael Gil'; gilmi@bezeqint.net
}

Received 20 July 2015; Accepted 31 August 2015

Academic Editor: William E. Fitzgibbon

Copyright (C) 2015 Michael Gil'. This is an open access article distributed under the Creative Commons Attribution License, which permits unrestricted use, distribution, and reproduction in any medium, provided the original work is properly cited.

We consider the equations $\sum_{k=0}^{m} c_{m-k} A^{k} X B^{k}=C$ and $\sum_{k=0}^{m} c_{m-k} A^{k} X B^{m-k}=C$, where $c_{k} \in \mathbb{C}(k=1, \ldots, m), c_{0}=1, A, B, C$ are given linear bounded operators in a Banach space and $X$ is to be found. Representations of solutions are derived. In the cases of Euclidean and Hilbert spaces, norm estimates for the solutions are suggested.

\section{Introduction and Statement of the Main Result}

The objects of this paper are the equations

$$
\begin{aligned}
\sum_{k=0}^{m} c_{m-k} A^{k} X B^{k} & =C, \\
\sum_{k=0}^{m} c_{m-k} A^{k} X B^{m-k} & =C,
\end{aligned}
$$

where $c_{0}=1, c_{k} \in \mathbb{C}(k=1, \ldots, m), A, B, C$ are given linear bounded operators in a Banach space $\mathscr{E}$ with a norm $\|\cdot\|$ and $X$ is to be found.

There is a long tradition of finding different expressions for the solution of an operator equation in the form of operator integrals and series, some prominent examples of which occur in the works of E. Heinz, M. G. Krein, M. Rosenblum and R. Bhatia, C. Davis, and A. McIntosh. A comprehensive summary of these is contained in [1] and [2]. This tradition was continued in [3], which deals with the equation

$$
\sum_{k=0}^{m} A^{m-k} X B^{k}=C .
$$

Clearly, (3) is a particular case of (2).

The special case

$$
A X-X B=C
$$

of (2) is the much studied Sylvester equation, of great interest in operator theory, numerical analysis, and engineering; compare [4-6] and references therein. Let $A^{*}$ be the operator adjoint to $A$. The equation

$$
A X+X A^{*}=C
$$

is the famous Lyapunov equation playing an essential role in the stability theory of differential equations. The equation

$$
X-A X B=C
$$

is an example of (1); in particular, the discrete Lyapunov equation

$$
X-A X A^{*}=C
$$

is an important tool in the theory of difference equations.

About other operator equations see [7-9] and references therein. In particular, [9] deals with necessary and sufficient conditions for the existence of solution to the systems of the general solution to a system of adjointable operator equations over Hilbert $C^{*}$ modules. In [8] the nonlinear operator equations of the form $A B A=A^{2}$ and $B A B=B^{2}$ are considered. It should be noted that in the finite dimensional case the numerical methods are well developed. Besides, the traditional methods convert matrix equations into their equivalent forms by using the Kronecker product, which involve the inversion of the associated large matrix and result in increasing computation and excessive computer memory. 
The recently suggested gradient based iterative methods are more powerful; compare [10-13] and references therein.

The aim of the present paper is to derive representations of solutions to (1) and (2) and to estimate the norms of these solutions. Such estimates are important, in particular, for the investigations of linear and perturbations of nonlinear differential and difference equations. Norm estimates for solutions of the Sylvester equation whose coefficients are normal operators can be found in [2]. In the finite dimensional case solution estimates for (4) and (6) have been established in [14].

In this paper, in particular, we considerably generalize the main results from [14].

Denote by $\sigma(A)$ and $r_{s}(A)$ the spectrum and spectral radius of $A$, respectively.

Theorem 1. Let $x_{k}(k=1, \ldots, m)$ be the roots taken with the multiplicities of the polynomial

$$
p(x):=\sum_{k=0}^{m} c_{k} x^{m-k}
$$

and let

$$
r_{s}(A) r_{s}(B)<\min _{k}\left|x_{k}\right|
$$

Then (1) has a unique solution $X$ which can be represented by

$$
\begin{aligned}
X & =(-1)^{m} \\
& \cdot \sum_{j_{1}, j_{2}, \ldots, j_{m}=0}^{\infty} \frac{1}{x_{1}^{j_{1}+1} x_{2}^{j_{2}+1} \cdots x_{m}^{j_{m}+1}} A^{j_{1}+j_{2}+\cdots+j_{m}} C B^{j_{1}+j_{2}+\cdots+j_{m}},
\end{aligned}
$$

and the series strongly converges.

The proof of this theorem is presented in the next section.

\section{Proof of Theorem 1}

Following [1, Section I.3], introduce the operators $A_{l}$ and $B_{r}$ by $A_{l} X:=A X$ and $B_{r} X:=X B$, respectively. Besides, $A_{l}$ and $B_{r}$ commute.

Let $f(z, w)$ be a scalar function regular on $\sigma(A) \times \sigma(B)$. Define the operator valued function

$$
\begin{aligned}
& \Phi(f, A, B) \\
& \quad:=-\frac{1}{4 \pi^{2}} \int_{L_{B}} \int_{L_{A}} f(z, w) R_{z}\left(A_{l}\right) R_{w}\left(B_{r}\right) d w d z,
\end{aligned}
$$

where $L_{A}, L_{B}$ are closed Jordan contours surrounding $\sigma(A)$ and $\sigma(B)$, respectively. So for any bounded linear operator $C$

$$
\begin{aligned}
\Phi & (f, A, B) C \\
& =-\frac{1}{4 \pi^{2}} \int_{L_{B}} \int_{L_{A}} f(z, w) R_{z}(A) C R_{w}(B) d w d z .
\end{aligned}
$$

Besides,

$$
\Phi\left(f_{1}, A, B\right) \Phi\left(f_{2}, A, B\right)=\Phi\left(f_{1} f_{2}, A, B\right),
$$

for functions $f_{1}, f_{2}$ regular on $\sigma(A) \times \sigma(B)$. Take $f(z, w)=$ $p(z w)$. If

$$
p(z w) \neq 0 \quad(z \in \sigma(A), w \in \sigma(B))
$$

then due to formula (3.5) from [1, Section I.3] (1) can be written as

$$
\Phi(p(z w), A, B) X=C
$$

Since

$$
p(x)=\prod_{k=1}^{m}\left(x-x_{k}\right),
$$

making use of (13), we can write

$$
\begin{aligned}
& \Phi\left(z w-x_{1}, A, B\right) \\
& \quad . \Phi\left(z w-x_{2}, A, B\right) \cdots \Phi\left(z w-x_{m}, A, B\right) X=C .
\end{aligned}
$$

But $\Phi\left(z w-x_{k}, A, B\right)=A_{l} B_{r}-x_{k} I$. Thus,

$$
\prod_{k=1}^{m}\left(A_{l} B_{r}-x_{k} I\right) X=C
$$

and therefore we get the following result.

Lemma 2. Under condition (14) equation (1) has a unique solution defined by

$$
X=\prod_{k=1}^{m}\left(A_{l} B_{r}-x_{k} I\right)^{-1} C .
$$

Furthermore, for $a \in \mathbb{C}$, satisfying

$$
|a|>r_{s}(A) r_{s}(B)
$$

consider the operator

$$
Z=-\sum_{k=0}^{\infty} \frac{1}{a^{k+1}} A_{l}^{k} B_{r}^{k} C=-\sum_{k=0}^{\infty} \frac{1}{a^{k+1}} A^{k} C B^{k} .
$$

The series converges and

$$
\begin{aligned}
A_{l} B_{r} Z & =A Z B=-\sum_{k=0}^{\infty} \frac{1}{a^{k+1}} A^{k+1} C B^{k+1} \\
& =-\sum_{k=0}^{\infty} \frac{1}{a^{k}} A^{k} C B^{k}+C=a Z+C .
\end{aligned}
$$

We thus get the following result.

Lemma 3. Let condition (20) hold. Then operator $Z$ defined by (21) is the unique solution to the equation $A Z B-a Z=C$ and $Z=\left(A_{l} B_{r}-a I\right)^{-1} C$. 
Proof of Theorem 1. Put

$$
\begin{gathered}
Y_{1}=\left(A_{l} B_{r}-x_{1} I\right)^{-1} C, \\
Y_{2}=\left(A_{l} B_{r}-x_{2} I\right)^{-1} Y_{1}, \\
\vdots \\
Y_{k}=\left(A_{l} B_{r}-x_{k} I\right)^{-1} Y_{k-1} .
\end{gathered}
$$

Lemma 2 implies $X=Y_{m}$. Due to the previous lemma $Y_{k}$ are solutions to the equations

$$
\begin{aligned}
A Y_{k} B-x_{k} Y_{k} & =Y_{k-1} \quad(k=2, \ldots, m), \\
A Y_{1} B-x_{1} Y_{1} & =C \\
Y_{j} & =-\sum_{k=0}^{\infty} \frac{1}{x_{j}^{k+1}} A^{k} Y_{k-1} B^{k},
\end{aligned}
$$

provided (9) holds. So

$$
\begin{aligned}
Y_{1} & =-\sum_{k=0}^{\infty} \frac{1}{x_{1}^{k+1}} A^{k} C B^{k} \\
Y_{2} & =-\sum_{j=0}^{\infty} \frac{1}{x_{2}^{j+1}} A^{j} Y_{1} B^{j}=\sum_{j=0}^{\infty} \frac{1}{x_{2}^{j+1}} A^{j} \sum_{k=0}^{\infty} \frac{1}{x_{1}^{k+1}} A^{k} C B^{k} B^{j} \\
& =\sum_{j, k=0}^{\infty} \frac{1}{x_{1}^{k+1} x_{2}^{j+1}} A^{k+j} C B^{k+j} .
\end{aligned}
$$

Continuing this process for $j=3, \ldots, m$, according to Lemma 2, we prove the theorem.

\section{Representations of Solutions to (2)}

Assume that $B$ is invertible; then from (2) we have

$$
\sum_{k=0}^{m} c_{m-k} A^{k} X B^{-k}=\widehat{C}:=C B^{-m} .
$$

Let $r_{l}(B)$ denote the lower spectral radius of $B: r_{l}(B)=$ $\min _{\lambda \in \sigma(B)}|\lambda|$. Taking into account the fact that $r_{l}(B)=$ $1 / r_{s}\left(B^{-1}\right)$ and applying now Theorem 1 to $(26)$ with $B^{-1}$ instead of $B$, we get the following result.

Corollary 4. Let

$$
r_{s}(A)<r_{l}(B) \min _{k}\left|x_{k}\right|
$$

Then (2) has a unique solution $X$ which can be represented by

$$
\begin{aligned}
X & =(-1)^{m} \\
& \cdot \sum_{j_{1}, j_{2}, \ldots, j_{m}=0}^{\infty} \frac{1}{x_{1}^{j_{1}+1} x_{2}^{j_{2}+1} \cdots x_{m}^{j_{m}+1}} A^{j_{1}+j_{2}+\cdots+j_{m}} C B^{-m-j_{1}-j_{2}-\cdots-j_{m}},
\end{aligned}
$$

and the series strongly converges.
Condition (27) does not allow us to consider the Lyapunov equation, since $r_{s}(A)=r_{s}\left(A^{*}\right)$. Because of this we are going to derive the representation of solutions to (2) under other conditions. To this end put

$$
\begin{aligned}
& \alpha(A)=\sup \operatorname{Re} \sigma(A), \\
& \beta(A)=\inf \operatorname{Re} \sigma(A) .
\end{aligned}
$$

According to (19) for a solution to (26) we have

$$
X=\prod_{k=1}^{m}\left(A_{l} B_{r}^{-1}-x_{k} I\right)^{-1} \widehat{C},
$$

provided

$$
p\left(\frac{z}{w}\right) \neq 0 \quad(z \in \sigma(A), w \in \sigma(B)) .
$$

Here $B_{r}^{-1}$ is defined as $B_{r}^{-1} C=C B^{-1}$. Thus

$$
\begin{aligned}
X & =\prod_{k=1}^{m}\left(A_{l} B_{r}^{-1}-x_{k} I\right)^{-1} B_{r}^{-m} B_{r}^{m} \widehat{C} \\
& =\prod_{k=1}^{m}\left(A_{l}-x_{k} B_{r}\right)^{-1} C .
\end{aligned}
$$

Now the invertibility condition for $B$ can be removed. We thus have proved the following result.

Lemma 5. Let the condition

$$
z-x_{k} w \neq 0 \quad(k=1, \ldots, m ; z \in \sigma(A), w \in \sigma(B))
$$

hold. Then (2) has a unique solution $X$ which can be represented by (32).

Lemma 6. Let the condition

$$
\beta(A)>\alpha(B)
$$

hold. Then (4) has a unique solution $X_{S}$, which can be represented as

$$
X_{S}=\int_{0}^{\infty} e^{-A t} C e^{B t} d t .
$$

Proof. For the brevity put $b=\alpha(B)$ and $c=(\beta(A)-\alpha(B)) / 2$. Then $\beta(A)=b+2 c$ and $\alpha(a B-I(b+c))=-c$. Moreover, $\alpha(-(A-I(b+c)))=\alpha(-A)+b+c=-\beta(A)+b+c=-c$. Equation (4) is equivalent to the following one:

$$
(A-(\alpha(B)+c) I) X-X(B-(\alpha(B)+c) I)=C .
$$

Due to Theorem 9.2 from [2] a solution of (36) is defined by the equality

$$
\begin{aligned}
X_{S} & =\int_{0}^{\infty} e^{-(A-I(\alpha(B)+c)) t} C e^{(B-(\alpha(B)+c) I) t} d t \\
& =\int_{0}^{\infty} e^{-A t} C e^{B t} d t,
\end{aligned}
$$

as claimed. 
Corollary 7. Let the condition

$$
\beta(A)>\alpha(a B) \quad(a \in \mathbb{C})
$$

hold. Then the equation

$$
A X-a X B=C
$$

has a unique solution $X_{a}$, which can be represented as

$$
X_{a}=\int_{0}^{\infty} e^{-A t} C e^{a B t} d t
$$

A solution of (39) is also given by $X_{a}=\left(A_{l}-a B_{r}\right)^{-1} C$. So under condition (38) we have

$$
\left(A_{l}-a B_{r}\right)^{-1} C=\int_{0}^{\infty} e^{-A t} C e^{a B t} d t .
$$

Now assume that

$$
\beta(A)>\max _{k} \alpha\left(x_{k} B\right) .
$$

Then by (41)

$$
\left(A_{l}-x_{k} B_{r}\right)^{-1}=\int_{0}^{\infty} e^{-A t} C e^{x_{k} B t} d t .
$$

Let $x_{k}=a_{k}+i b_{k}$. Then $\alpha\left(x_{k} B\right)=\sup _{s \in \sigma(B)}\left(a_{k} \operatorname{Re} s-b_{k} \operatorname{Im} s\right)$. So condition (42) means that $\beta(A)>\sup _{s \in \sigma(B)}\left(a_{k} \operatorname{Re} s-b_{k} \operatorname{Im} s\right)$.

Put

$$
\begin{gathered}
W_{1}=\left(A_{l}-x_{1} B_{r}\right)^{-1} C, \\
W_{2}=\left(A_{l}-x_{2} B_{r}\right)^{-1} Y_{1}, \\
\vdots \\
W_{k}=\left(A_{l}-x_{k} B_{r}\right)^{-1} W_{k-1} .
\end{gathered}
$$

Then $X=W_{m}$. Due to the previous lemma $Y_{k}$ are solutions to the equations

$$
\begin{aligned}
A W_{k}-x_{k} B & =W_{k-1} \quad(k=2, \ldots, m), \\
A Y_{1} B-x-1 Y_{1} & =C, \\
W_{j} & =-\int_{0}^{\infty} e^{-A t} W_{j-1} e^{x_{k} B t} d t .
\end{aligned}
$$

provided (42) holds. So

$$
\begin{aligned}
W_{1} & =-\int_{0}^{\infty} e^{-A t_{1}} C e^{x_{1} B t_{1}} d t_{1}, \\
W_{2} & =-\int_{0}^{\infty} e^{-A t_{2}} W_{1} e^{x_{2} B t_{2}} d t_{2} \\
& =\int_{0}^{\infty} \int_{0}^{\infty} e^{-\left(t_{1}+t_{2}\right) A} C e^{\left(x_{2} t_{2}+x_{1} t_{1}\right) B} d t_{1} d t_{2} .
\end{aligned}
$$

Continuing this process for $j=3, \ldots, m$, according to Lemma 5, we obtain

$X$

$$
=\int_{0}^{\infty} \cdots \int_{0}^{\infty} e^{-\left(t_{1}+\cdots+t_{m}\right) A} C e^{\left(x_{1} t_{1}+\cdots+x_{m} t_{m}\right) B} d t_{1} \cdots d t_{m} .
$$

Theorem 8. Let $x_{k}(k=1, \ldots, m)$ be the roots of $p(x)$ and let condition (42) hold. Then (2) has a unique solution $X$, which can be represented by (47).

\section{Solution Estimates in the Finite Dimensional Case}

4.1. Equation (1). In this section $\mathscr{E}=\mathbb{C}^{n}$ is a Euclidean space. Let $\lambda_{k}(A)(k=1, \ldots, n)$ be the eigenvalues of a matrix $A$ counted with their multiplicities. The following quantity (the departure from normality of $A$ ) plays a key role hereafter:

$$
g(A)=\left[N_{2}(A)^{2}-\sum_{k=1}^{n}\left|\lambda_{k}(A)\right|^{2}\right]^{1 / 2}
$$

where $N_{2}(A)=\left(\text { Trace } A A^{*}\right)^{1 / 2}$ is the Frobenius (HilbertSchmidt norm) of $A$.

The following relations are checked in [15, Section 2.1]:

$$
\begin{aligned}
& g^{2}(A) \leq N_{2}(A)^{2}(A)-\left|\operatorname{Trace} A^{2}\right| \\
& g^{2}(A) \leq \frac{N_{2}^{2}\left(A_{I}\right)}{2}
\end{aligned}
$$

where $A_{I}=\left(A-A^{*}\right) / 2 i$. If $A$ is a normal matrix: $A A^{*}=$ $A^{*} A$, then $g(A)=0$. If $A_{1}$ and $A_{2}$ are commuting matrices, then $g\left(A_{1}+A_{2}\right) \leq g\left(A_{1}\right)+g\left(A_{2}\right)$. By the inequality between geometric and arithmetic mean values we have

$$
\left(\frac{1}{n} \sum_{k=1}^{n}\left|\lambda_{k}(A)\right|^{2}\right)^{n} \geq\left(\prod_{k=1}^{n}\left|\lambda_{k}(A)\right|\right)^{2}
$$

So $g^{2}(A) \leq N_{2}(A)^{2}-n(\operatorname{det} A)^{2 / n}$. Obviously $g(a A)=$ $|a| g(A)(a \in \mathbb{C})$.

By Corollary 2.7.2 from [15], one has

$$
\left\|A^{w}\right\| \leq \sum_{k=0}^{n-1} \frac{w ! g^{k}(A) r_{s}^{w-k}(A)}{(w-k) !(k !)^{3 / 2}} \quad(w=1,2, \ldots) .
$$

Note that $1 /(w-k) !=0(s<k)$. Furthermore, due to Example 1.10.3 from [15] we have

$$
\left\|e^{A t}\right\| \leq e^{\alpha(A) t} \sum_{k=0}^{n-1} \frac{t^{k} g^{k}(A)}{(k !)^{3 / 2}} \quad(t \geq 0) .
$$

From (21) and Lemma 3 we have

$$
\left(A_{l} B_{r}-a I\right)^{-1} C=-\sum_{w=0}^{\infty} \frac{1}{a^{w+1}} A^{w} C B^{w}
$$

$$
(s=1, \ldots, m) \text {, }
$$

provided condition (20) holds. Hence, and from (51), it directly follows

$$
\left\|\left(A_{l} B_{r}-x_{s} I\right)^{-1}\right\| \leq J\left(A, B, x_{s}\right),
$$


where

$$
\begin{aligned}
J(A, B, a)= & \sum_{j, k=0}^{n-1} \frac{g^{k}(A) g^{j}(B)}{(k ! j !)^{3 / 2}} \\
& \cdot \sum_{w=0}^{\infty} \frac{1}{|a|^{w+1}} \frac{(w !)^{2} r_{s}^{w-k}(A) r_{s}^{w-j}(B)}{(w-k) !(w-j) !} .
\end{aligned}
$$

Now Lemma 2 and (54) imply the following result.

Corollary 9. Let condition (9) hold. Then a unique solution X to (1) in $\mathbb{C}^{n}$ satisfies the inequality

$$
\|X\| \leq\|C\| J\left(A, B, x_{1}\right) J\left(A, B, x_{2}\right) \cdots J\left(A, B, x_{m}\right) .
$$

If $A$ is normal, then $g(A)=0$ and

$$
J(A, B, a)=\sum_{j=0}^{n-1} \frac{g^{j}(B)}{(j !)^{3 / 2}} \sum_{w=0}^{\infty} \frac{1}{|a|^{w+1}} \frac{w ! r_{s}^{w}(A) r_{s}^{w-j}(B)}{(w-j) !} .
$$

But

$$
\begin{aligned}
& \sum_{w=0}^{\infty} \frac{w ! r_{s}^{w}(A) r_{s}^{w-j}(B)}{|a|^{w-j}(w-j) !} \\
& =\frac{r_{s}^{j}(A)}{|a|^{j+1}} \sum_{w=0}^{\infty} \frac{w !\left(r_{s}(A) r_{s}(B)\right)^{w-j}}{|a|^{w-j}(w-j) !} \\
& =\frac{r_{s}^{j}(A)}{|a|^{j+1}} \frac{d^{j}}{d x^{j}} \sum_{w=0}^{\infty} x^{w}=j ! \frac{r_{s}^{j}(A)}{|a|^{j+1}}(1-x)^{-j-1} \\
& \qquad\left(x=\frac{1}{|a|} r_{s}(A) r_{s}(B)\right) .
\end{aligned}
$$

Thus, we have

$$
J(A, B, a) \leq \sum_{j=0}^{n-1} \frac{g^{j}(B) r_{s}^{j}(A)}{(j !)^{1 / 2}\left(|a|-r_{s}(A) r_{s}(B)\right)^{j+1}},
$$

provided $A$ is normal. If both $A$ and $B$ are normal, then

$$
J(A, B, a) \leq \frac{1}{|a|-r_{s}(A) r_{s}(B)} .
$$

Furthermore, obviously,

$$
\begin{aligned}
\sum_{w=0}^{\infty} \frac{(w !)^{2} b^{2 w}}{(w-k) !(w-j) !} & \leq \sum_{t=0}^{\infty} \frac{t ! b^{t}}{(t-k) !} \sum_{w=0}^{\infty} \frac{w ! b^{w}}{(w-j) !} \\
& =b^{k+j} \sum_{t=0}^{\infty} \frac{t ! b^{t-k}}{(t-k) !} \sum_{w=0}^{\infty} \frac{w ! b^{w-j}}{(w-j) !} \\
& =\frac{j ! k ! b^{k+j}}{(1-b)^{k+1}(1-b)^{j+1}} \\
& (0<b<1) .
\end{aligned}
$$

Taking

$$
b=b(A, B, a)=\sqrt{\frac{r_{s}(A) r_{s}(B)}{|a|}},
$$

we get

$$
J(A, B, a) \leq \widehat{J}(A, B, a),
$$

where

$$
\begin{aligned}
& \widehat{J}(A, B, a)=\frac{1}{|a|} \\
& \quad \cdot \sum_{j, k=0}^{n-1} \frac{g^{k}(A) g^{j}(B) b^{k+j}(A, B, a)}{(k ! j !)^{1 / 2}(1-b(A, B, a))^{k+1}(1-b(A, B, a))^{j+1}} .
\end{aligned}
$$

Now Corollary 9 implies the following result.

Corollary 10. Let condition (9) hold. Then a unique solution $X$ of (1) in $\mathbb{C}^{n}$ satisfies the inequality

$$
\|X\| \leq\|C\| \widehat{J}\left(A, B, x_{1}\right) \widehat{J}\left(A, B, x_{2}\right) \cdots \widehat{J}\left(A, B, x_{m}\right) .
$$

Theorem 1 and simple calculations imply the following result.

Corollary 11. Let $r_{s}(A)<1$. Then (7) has a unique solution $X_{L}$, which satisfies the inequalities

$$
\left\|X_{L}\right\| \leq\|C\| \sum_{m=0}^{\infty}\left(\sum_{k=0}^{n-1} \frac{g^{k}(A) m ! r_{s}^{m-k}(A)}{(k !)^{3 / 2}(m-k) !}\right)^{2} .
$$

From Corollary 11 and (63) it follows that

$$
\left\|X_{L}\right\| \leq\|C\|\left(\sum_{k=0}^{n-1} \frac{g^{k}(A)}{(k !)^{1 / 2}\left(1-r_{s}(A)\right)^{k+1}}\right)^{2} .
$$

4.2. Equation (2). Due to (41) and (52) under (38) we have

$$
\begin{aligned}
\left\|\left(A_{l}-a B_{r}\right)^{-1}\right\| & \leq \int_{0}^{\infty}\left\|e^{-A s}\right\|\left\|e^{a B s}\right\| d s \\
& \leq \int_{0}^{\infty} e^{-s c} \sum_{j, k=0}^{n-1} \frac{s^{k+j} g^{k}(A) g^{j}(a B)}{(k ! j !)^{3 / 2}} d s,
\end{aligned}
$$

where $c=\beta(A)-\alpha(a B)$. But

$$
\int_{0}^{\infty} s^{k+j} e^{-c s} d s=\frac{(k+j) !}{c^{k+j+1}} .
$$

Thus

$$
\left\|\left(A_{l}-a B_{r}\right)^{-1}\right\| \leq \gamma(a, A, B),
$$

where

$$
\gamma(a, A, B):=\sum_{j, k=0}^{n-1} \frac{(k+j) ! g^{k}(A) g^{j}(a B)}{(\beta(A)-\alpha(a B))^{k+j+1}(k ! j !)^{3 / 2}},
$$

provided $\beta(A)>\alpha(a B)$. Due to Lemma 5 we arrive at the following result.

Corollary 12. Let condition (42) hold. Then a unique solution $X$ to (2) in $\mathbb{C}^{n}$ satisfies the inequality

$$
\|X\| \leq\|C\| \gamma\left(A, B, x_{1}\right) \gamma\left(A, B, x_{2}\right) \cdots \gamma\left(A, B, x_{m}\right) .
$$


If $A$ is normal, then $g(A)=0$ and therefore

$$
\gamma(a, A, B):=\sum_{j=0}^{n-1} \frac{|a|^{j} g^{j}(B)}{(\beta(A)-\alpha(a B))^{j+1}(j !)^{1 / 2}} .
$$

If both $A$ and $B$ are normal, then

$$
\gamma(a, A, B) \leq \frac{1}{\beta(A)-\alpha(a B)} .
$$

For the Sylvester equation we have the following result.

Corollary 13. Let $\alpha(B)<\beta(A)$. Then (4) in $\mathbb{C}^{n}$ has a unique solution $Y_{S}$, which satisfies the inequality

$$
\left\|Y_{S}\right\| \leq \sum_{j, k=0}^{n-1} \frac{(k+j) ! g^{k}(A) g^{j}(B)}{(\beta(A)-\alpha(B))^{k+j+1}(k ! j !)^{3 / 2}} .
$$

Consider the Lyapunov equation (5). Taking in Corollary $13-A$ instead of $A$ and $A^{*}$ instead of $B$, since $\alpha\left(A^{*}\right)=\alpha(A)$ and $\beta(-A)=-\alpha(A)$, we get the following result.

Corollary 14. Let $\alpha(A)<0$. Then (5) in $\mathbb{C}^{n}$ has a unique solution $Y_{L}$, which satisfies the inequality

$$
\left\|Y_{L}\right\| \leq\|C\| \sum_{j, k=0}^{n-1} \frac{(k+j) ! g^{k+j}(A)}{(2|\alpha(A)|)^{k+j+1}(k ! j !)^{3 / 2}} .
$$

\section{Solution Estimates in \\ the Infinite-Dimensional Case}

5.1. Equation (1). In this section $\mathscr{E}=H$, a separable Hilbert space. It is assumed that

$$
A-A^{*} \text { and } B-B^{*} \text { are Hilbert-Schmidt operators. }
$$

Put

$$
g_{I}(A)=\left(\frac{1}{2} N_{2}^{2}\left(A-A^{*}\right)-2 \sum_{k=1}^{\infty}\left|\operatorname{Im} \lambda_{k}(A)\right|^{2}\right)^{1 / 2} .
$$

Recall that $N_{2}(A)=\left(\text { Trace } A A^{*}\right)^{1 / 2}$ and $\lambda_{k}(A)$ are the eigenvalues of a matrix $A$ counted with their multiplicities. If $A$ is a Hilbert-Schmidt operator, then $g_{I}(A)=g(A)$, where

$$
g(A)=\left(N_{2}^{2}\left(A-A^{*}\right)-\sum_{k=1}^{\infty}\left|\lambda_{k}(A)\right|^{2}\right)^{1 / 2} ;
$$

compare Lemma 6.5.2 [15]. Due to Example 7.10.2 from [15] we can write

$$
\left\|A^{w}\right\| \leq \sum_{k=0}^{\infty} \frac{w ! g_{I}^{k}(A) r_{s}^{w-k}(A)}{(w-k) !(k !)^{3 / 2}} \quad(w=1,2, \ldots) .
$$

As it was shown in Section 4

$$
\begin{array}{r}
\left(A_{l} B_{r}-x_{s} I\right)^{-1} C=-\sum_{w=0}^{\infty} \frac{1}{x_{s}^{w+1}} A^{w} C B^{w} \\
\quad(s=1, \ldots, m),
\end{array}
$$

provided (9) holds. Hence, and from (80), it directly follows that

$$
\left\|\left(A_{l} B_{r}-x_{s} I\right)^{-1}\right\| \leq J_{H}\left(A, B, x_{k}\right),
$$

where

$$
\begin{aligned}
J_{H}(A, B, a)= & \sum_{j, k=0}^{\infty} \frac{g_{I}^{k}(A) g_{I}^{j}(B)}{(k ! j !)^{3 / 2}} \\
& \cdot \sum_{w=0}^{\infty} \frac{1}{|a|^{w+1}} \frac{(w !)^{2} r_{s}^{w-k}(A) r_{s}^{w-j}(B)}{(w-k) !(w-j) !} .
\end{aligned}
$$

Now Lemma 2 implies the following result.

Corollary 15. Let conditions (9) and (77) hold. Then the unique solution $X$ to (1) in $H$ satisfies the inequality

$\|X\|$

$$
\leq\|C\| J_{H}\left(A, B, x_{1}\right) J_{H}\left(A, B, x_{2}\right) \cdots J_{H}\left(A, B, x_{m}\right) .
$$

If $A$ is normal, then $g\left(A_{I}\right)=0$ and according to (59)

$$
J_{H}(A, B, a) \leq \sum_{j=0}^{\infty} \frac{g_{I}^{j}(B) r_{s}^{j}(A)}{(j !)^{1 / 2}\left(|a|-r_{s}(A) r_{s}(B)\right)^{j+1}} .
$$

If both $A$ and $B$ are normal, then

$$
J_{H}(A, B, a) \leq \frac{1}{|a|-r_{s}(A) r_{s}(B)} .
$$

Furthermore, according to (63), we get $J_{H}(A, B, a) \leq \widehat{J}_{H}(A$, $B, a)$, where

$$
\begin{aligned}
& \widehat{J}_{H}(A, B, a)=\frac{1}{|a|} \\
& \cdot \sum_{j, k=0}^{\infty} \frac{g_{I}^{k}(A) g_{I}^{j}(B) b^{k+j}(A, B, a)}{(k ! j !)^{1 / 2}(1-b(A, B, a))^{k+1}(1-b(A, B, a))^{j+1}},
\end{aligned}
$$

where $b(A, B, a)$ is defined by (62). Now Corollary 15 implies the following result.

Corollary 16. Let conditions (9) and (77) hold. Then a unique solution $X$ of (1) in $H$ satisfies the inequality

$\|X\|$

$$
\leq\|C\| \widehat{J}_{H}\left(A, B, x_{1}\right) \widehat{J}_{H}\left(A, B, x_{2}\right) \cdots \widehat{J}_{H}\left(A, B, x_{m}\right) .
$$

By virtue of Corollary 15 we can assert that a unique solution $X_{L}$ of (7) satisfies the inequality

$$
\left\|X_{L}\right\| \leq\|C\| \sum_{m=0}^{\infty}\left(\sum_{k=0}^{\infty} \frac{g_{I}^{k}(A) m ! r_{s}^{m-k}(A)}{(k !) \frac{3}{2}(m-k) !}\right)^{2},
$$

provided $A$ satisfies conditions $(77)$ and $r_{s}(A)<1$. From Corollary 16 it follows that

$$
\left\|X_{L}\right\| \leq\|C\|\left(\sum_{k=0}^{\infty} \frac{g_{I}^{k}(A)}{(k !)^{1 / 2}\left(1-r_{s}(A)\right)^{k+1}}\right)^{2} .
$$


5.2. Equation (2). Due to Example 7.10.3 from [15] we have

$$
\left\|e^{a A t}\right\| \leq e^{\alpha(a A) t} \sum_{k=0}^{\infty} \frac{t^{k}|a|^{k} g^{k}\left(A_{I}\right)}{(k !)^{3 / 2}} \quad(a \in \mathbb{C}, t \geq 0),
$$

provided $A$ satisfies (77). Now let us consider (2), assuming that condition (42) holds. Due to (41) and (91),

$$
\begin{aligned}
\left\|\left(A_{l}-a B_{r}\right)^{-1}\right\| & \leq \int_{0}^{\infty}\left\|e^{-A s}\right\|\left\|e^{a B s}\right\| d s \\
& \leq \int_{0}^{\infty} e^{-s c} \sum_{j, k=0}^{\infty} \frac{s^{k+j} g_{I}^{k}(A) g_{I}^{j}(a B)}{(k ! j !)^{3 / 2}} d s,
\end{aligned}
$$

where $c=\beta(A)-\alpha(a B)$. Thus

$$
\left\|\left(A_{l}-a B_{r}\right)^{-1}\right\| \leq \gamma_{H}(A, B, a),
$$

where

$$
\gamma_{H}(a, A, B):=\sum_{j, k=0}^{\infty} \frac{(k+j) ! g_{I}^{k}(A) g_{I}^{j}(a B)}{(\beta(A)-\alpha(a B))^{k+j+1}(k ! j !)^{3 / 2}},
$$

provided $\beta(A)>\alpha(a B)$. Due to Lemma 5 we arrive at the following result.

Corollary 17. Let conditions (42) and (77) hold. Then the unique solution $X$ to (2) in $H$ satisfies the inequality

\section{$\|X\|$}

$$
\leq\|C\| \gamma_{H}\left(A, B, x_{1}\right) \gamma_{H}\left(A, B, x_{2}\right) \cdots \gamma_{H}\left(A, B, x_{m}\right) \text {. }
$$

Let $\beta(A)>\alpha(a B)$. Then, if $A$ is normal, then $g_{I}(A)=0$ and therefore

$$
\gamma_{H}(a, A, B):=\sum_{j=0}^{\infty} \frac{|a|^{j} g_{I}^{j}(B)}{(\beta(A)-\alpha(a B))^{j+1}(j !)^{1 / 2}} .
$$

If both $A$ and $B$ are normal, then

$$
\gamma_{H}(a, A, B)=\frac{1}{\beta(A)-\alpha(a B)} .
$$

According to Corollary 17 we get the following result.

Corollary 18. Let $A$ satisfies conditions (77) and $\alpha(A)<0$. Then the solution $Y_{L}$ to the Lyapunov equation (5) in $H$ satisfies the inequality

$$
\left\|Y_{l}\right\| \leq\|C\| \sum_{j, k=0}^{\infty} \frac{(k+j) ! g_{I}^{k+j}(A)}{(2|\alpha(A)|)^{k+j+1}(k ! j !)^{3 / 2}} .
$$

\section{References}

[1] Y. L. Daleckii and M. G. Krein, Stability of Solutions of Differential Equations in Banach Space, American Mathematical Society, Providence, RI, USA, 1971.

[2] R. Bhatia and P. Rosenthal, "How and why to solve the operator equation $\mathrm{AX}-\mathrm{XB}=Y$," The Bulletin of the London Mathematical Society, vol. 29, no. 1, pp. 1-21, 1997.

[3] R. Bhatia and M. Uchiyama, "The operator equation $\sum_{i=1}^{n} A^{n-1} X B^{i}=Y$," Expositiones Mathematicae, vol. 27, no. 3, pp. 251-255, 2009.

[4] M. Dehghan and M. Hajarian, "The reflexive and anti-reflexive solutions of a linear matrix equation and systems of matrix equations," Rocky Mountain Journal of Mathematics, vol. 40, no. 3, pp. 825-848, 2010.

[5] M. Konstantinov, D.-W. Gu, V. Mehrmann, and P. Petkov, Perturbation Theory for Matrix Equations, vol. 9 of Studies in Computational Mathematics, North-Holland, Amsterdam, The Netherlands, 2003.

[6] A. G. Mazko, "Matrix equations, spectral problems and stability of dynamic systems," in Stability, Oscillations and Optimization of Systems, Cambridge Scientific Publishers, 2008.

[7] D. S. Djordjević, "Explicit solution of the operator equation $A^{*} X+X^{*} A=B$," Journal of Computational and Applied Mathematics, vol. 200, no. 2, pp. 701-704, 2007.

[8] B. P. Duggal, "Operator equations $A B A=A^{2}$ and $B A B=B^{2}$," Functional Analysis, Approximation and Computation, vol. 3, no. 1, pp. 9-18, 2011.

[9] Q.-W. Wang and C.-Z. Dong, "The general solution to a system of adjointable operator equations over Hilbert $C^{*}$ modules," Operators and Matrices, vol. 5, no. 2, pp. 333-350, 2011.

[10] B. Zhou, G.-R. Duan, and Z.-Y. Li, "Gradient based iterative algorithm for solving coupled matrix equations," Systems \& Control Letters, vol. 58, no. 5, pp. 327-333, 2009.

[11] F. Ding and T. Chen, "Gradient based iterative algorithms for solving a class of matrix equations," IEEE Transactions on Automatic Control, vol. 50, no. 8, pp. 1216-1221, 2005.

[12] L. Xie, J. Ding, and F. Ding, "Gradient based iterative solutions for general linear matrix equations," Computers \& Mathematics with Applications, vol. 58, no. 7, pp. 1441-1448, 2009.

[13] F. P. A. Beik, D. K. Salkuyeh, and M. M. Moghadam, "Gradientbased iterative algorithm for solving the generalized coupled Sylvester-transpose and conjugate matrix equations over reflexive (anti-reflexive) matrices," Transactions of the Institute of Measurement and Control, vol. 36, no. 1, pp. 99-110, 2014.

[14] M. I. Gil', "Norm estimates for solutions of matrix equations $A X-X B=C$ and $X-A X B=C$," Discussiones Mathematicae: Differential Inclusions, Control \& Optimization, vol. 34, no. 2, pp. 191-206, 2014.

[15] M. I. Gil', Operator Functions and Localization of Spectra, vol. 1830 of Lecture Notes in Mathematics, Springer, Berlin, Germany, 2003.

\section{Conflict of Interests}

The author declares that there is no conflict of interests regarding the publication of this paper. 


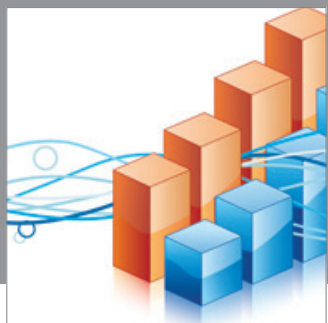

Advances in

Operations Research

mansans

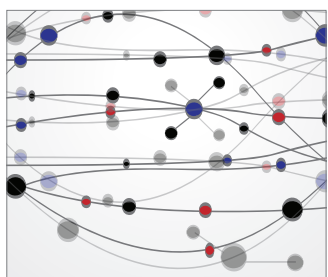

The Scientific World Journal
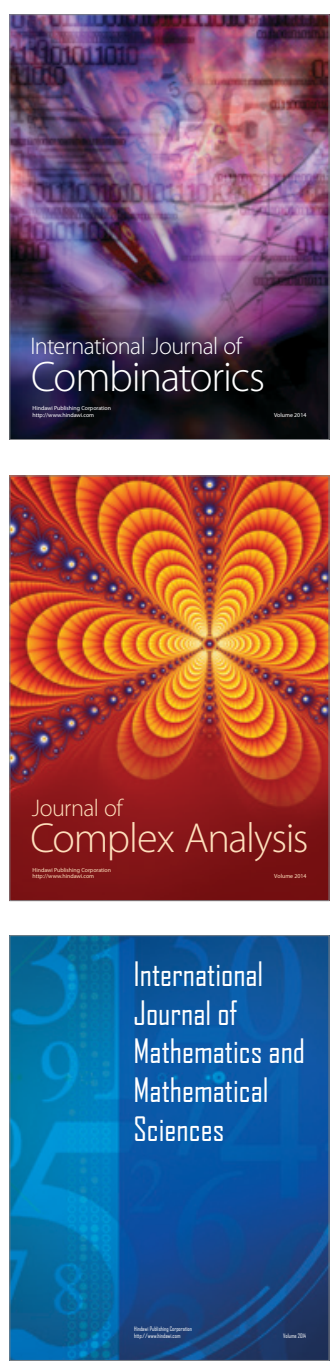
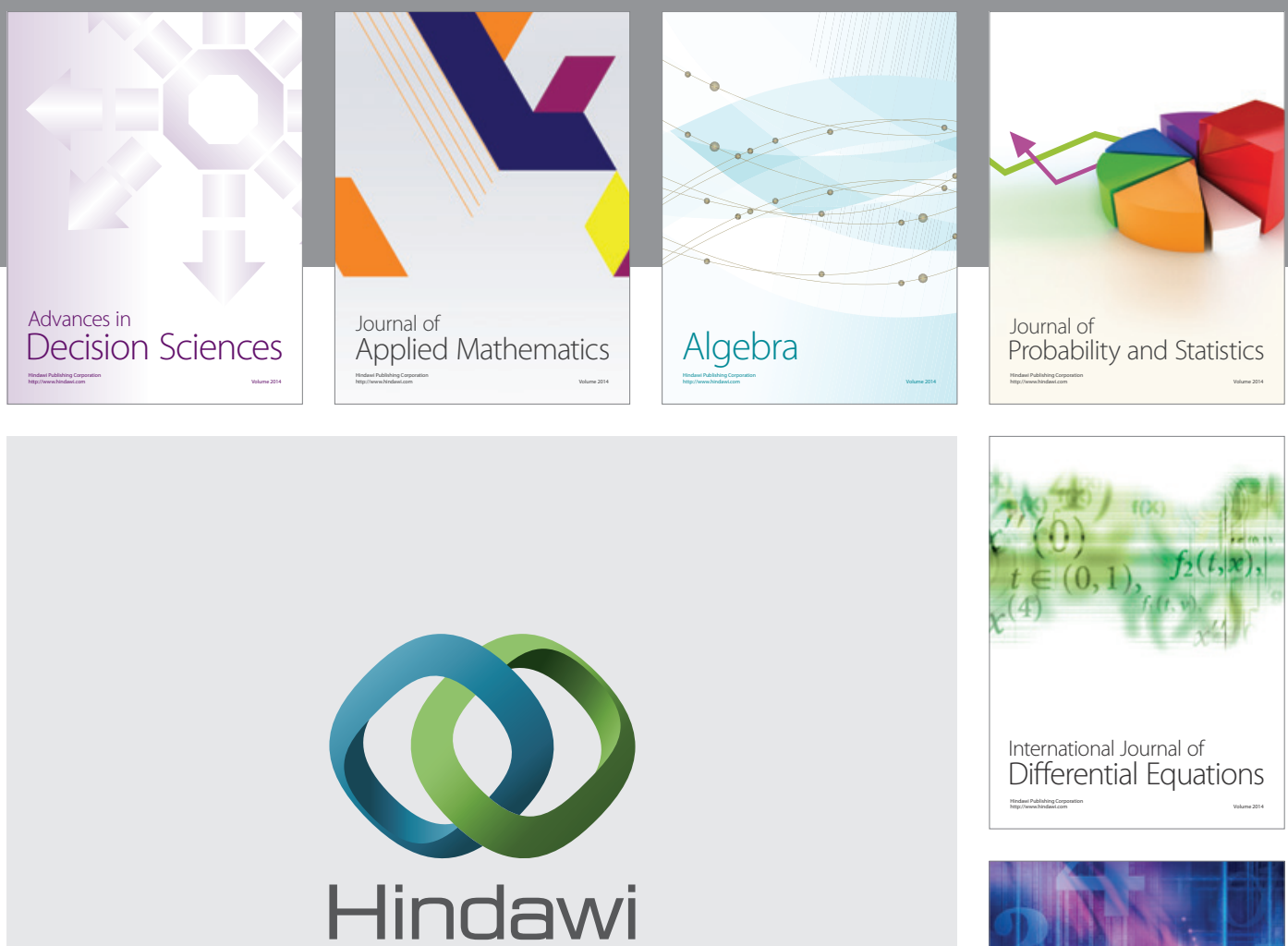

Submit your manuscripts at http://www.hindawi.com
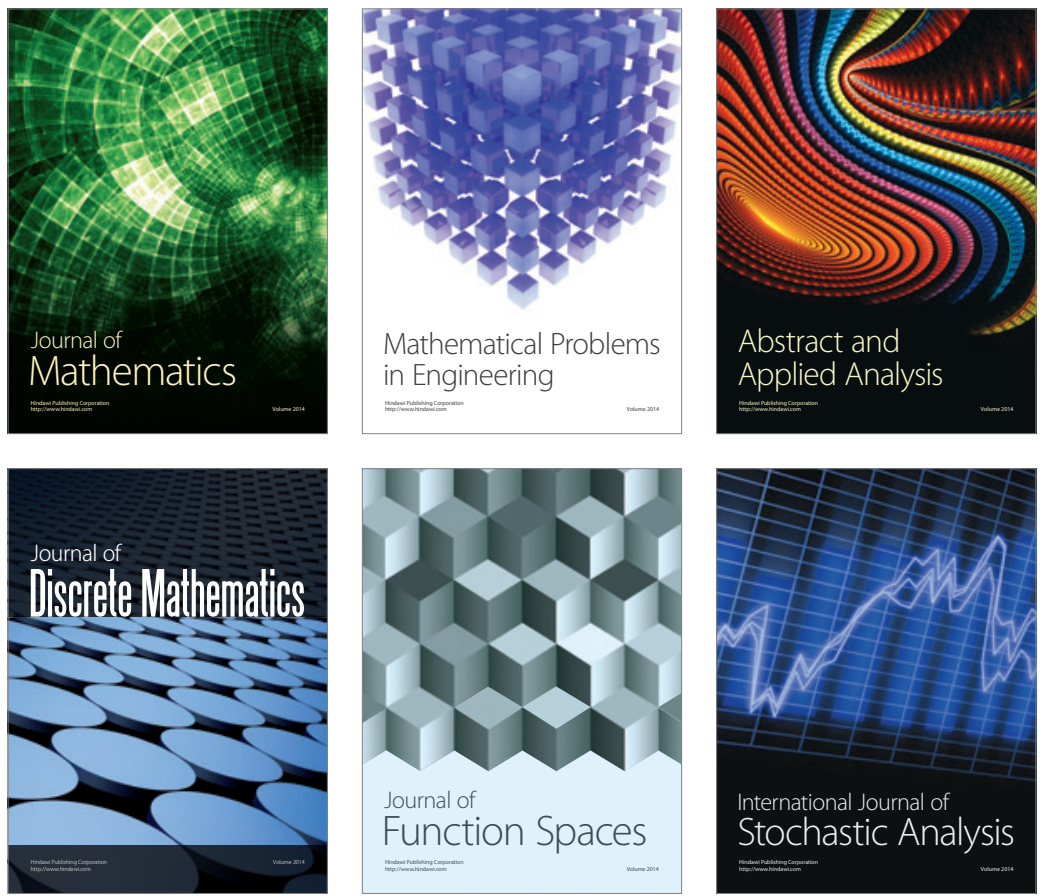

Journal of

Function Spaces

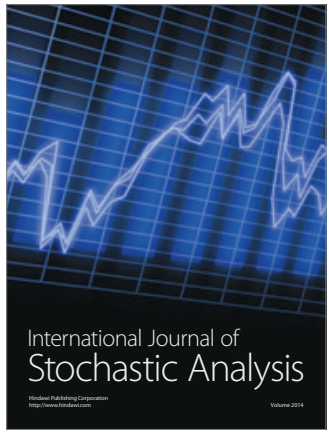

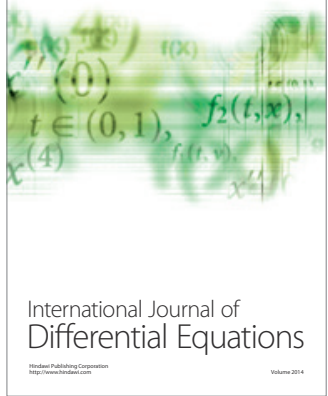
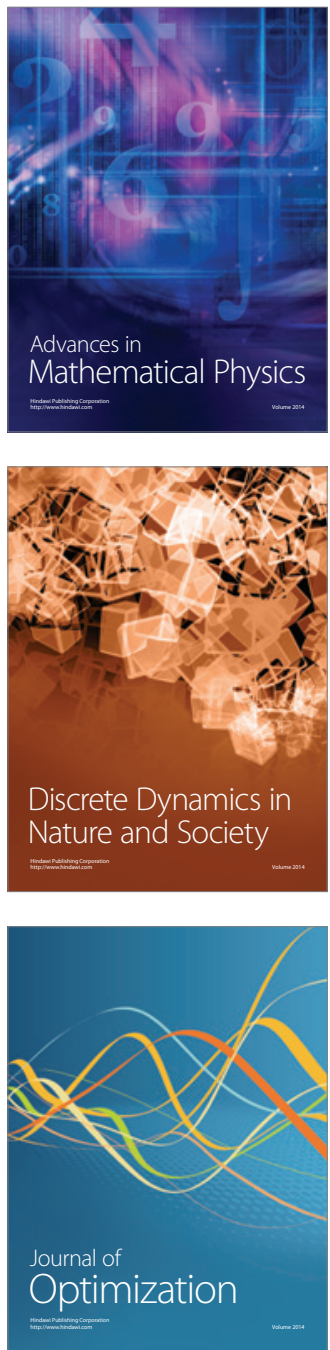